Pacific Journal of Mathematics

stR LLS CoNvERsES trieutratrat 


\title{
SUR LES CONVEXES UBIQUITAIRES
}

\author{
G. Coquet et J. C. Dupin
}

In this paper we develop and generalize some previously announced results concerning ubiquitous convex sets. We show two conditions which are sufficient to assert that a convex set which is ubiquitous-elementary or ubiquitous-basic is also linearly bounded. In showing the essential part played by the ubiquitous convex sets of countable infinite dimension we establish that in an infinite-dimensional real vector space every ubiquitous convex set contains a linearly bounded ubiquitous convex set of a specific type.

I. Introduction. Les convexes ubiquitaires ont été introduits et étudiés par Klee dans [5] et [6] et leur intérêt a été mis en évidence dans de nombreux articles du même auteur; l'existence dans tout espace vectoriel réel de dimension infinie, d'un convexe ubiquitaire linéairement borné a été remarquée et utilisée par Klee dans [6].

Dans [1] et [3], nous avons caractérisé les convexes ubiquitaires en tant que sur-ensembles de convexes ubiquitaires particuliers: les "convexes ubiquitaires-élémentaires" et les "convexes ubiquitairesbasiques".

Dans [4], nous avons énoncé deux critères permettant d'affirmer qu'un convexe ubiquitaire-élémentaire ou un convexe ubiquitaire-basique est linéairement borné et avons énoncé le résultat suivant: tout convexe ubiquitaire est sur-ensemble d'un convexe ubiquitaire linéairement borné.

Dans cet article, nous démontrons les critères précédents (Théorèmes 3.1 et 3.2) et généralisons le résultat dont il est question précédemment à partir du théorème suivant (Théorème 5.1): Dans un espace vectoriel réel de dimension infinie, tout convexe ubiquitaire contenant l'origine est sur-ensemble de l'enveloppe convexe d'une réunion de convexes qui sont tous de dimension infinie dénombrable et ubiquitaires dans les sous-espaces vectoriels qu'ils engendrent, la somme de ces sous-espaces vectoriels étant directe et égale à l'espace tout entier. De ce théorème, nous déduisons, en outre, un nouveau théorème de caractérisation des convexes ubiquitaires (Théorème 5.2) plus général que celui énoncé dans [4] et qui établit le lien étroit existant entre les convexes ubiquitaires et les convexes ubiquitaires linéairement bornés de dimension infinie dénombrable.

II. Rappels. Dans toute la suite les espaces vectoriels sont réels et les relations d'ordre sont notées $\leqq$. 
On désigne par $N$ l'ensemble des entiers $\geqq 0$, par $N^{*}$ l'ensemble des entiers $>0$ et par $\boldsymbol{R}_{+}^{*}$ l'ensemble des réels $>0$.

A étant une partie d'un espace vectoriel $E$, nous notons $S[A]$ le plus petit sous-espace vectoriel de $E$ contenant $A$ (si $S[A]$ est de dimension finie, $A$ est dite de dimension finie); nous notons $C[A]$ (resp.: $C S[A]$ ) l'enveloppe convexe (resp.: l'enveloppe convexe symétrique) de $A$; nous notons lin $A$ l'ensemble $A \cup$ lina $A$, lina $A$ désignant l'ensemble des points $x$ de $E$ qui sont linéairement accessibles de $A$ ( $x$ est dit linéairement accessible de $A$ s'il existe $a \in A, a \neq x$, tel que $[a, x[\subset A) ; A$ est dite ubiquitaire (resp.: proprement ubiquitaire) si lin $A=E$ (resp.: $\operatorname{lin} A=E$ et $A \neq E$ ); $A$ est dite linéairement bornée si son intersection avec toute droite est bornée (cette droite étant munie de la topologie naturelle), si $A$ est convexe, alors $A$ est linéairement bornée si et seulement si elle ne contient aucune demidroite.

Nous allons maintenant nous borner à rappeler les définitions et les résultats obtenus dans [1], [2] et [3] qui seront utiles pour la suite.

2.1. Notion de famille de décomposition.

2.1.1. DÉfinition (voir [1], paragraphe 3.1 ou [2], p. 899).

Soient $E$ un espace vectoriel de dimension infinie et $\mathscr{B}=\left(e_{i}\right)_{i \in I}$ une base bien ordonnée de $E$ (on suppose donc que $I$ est bien ordonné).

Soit $\left(f_{j}\right)_{j \in J}$ une famille de vecteurs (resp.: une base) de $E$ avec $f_{j}=\sum_{i \in I} a_{j, i} e_{i}$. Soit $r=\left(r_{i}\right)_{i \in I}$ un élément fixé de $\left(\boldsymbol{R}_{+}^{*}\right)^{I}$.

Posons, pour tout $i \in I$,

$$
\begin{aligned}
& P_{i}(r)=\left\{j\left|j \in J, \sum_{k>i}\right| a_{j, k} \mid \geqq r_{i}\right\}, \\
& Q_{i}(r)=\left\{v_{j, i} \mid j \in P_{i}(r), v_{j, i}=\frac{a_{j, i}}{\sum_{k>i}\left|a_{j, k}\right|}\right\} .
\end{aligned}
$$

Si, pour tout $i \in I, Q_{i}(r)$ n'est pas borné dans $\boldsymbol{R}$, on dit que $\left(f_{j}\right)_{j \in J}$ est une r-famille (resp.: r-base) de décomposition de $E$ associée à $\mathscr{B}$. $\mathrm{Si}$, pour tout $i \in I, Q_{i}(r)$ n'est ni majoré ni minoré dans $\boldsymbol{R}$, on dit que $\left(f_{j}\right)_{j \in J}$ est une $r$-famille (resp.: $r$-base) de décomposition forte de $E$ associée à $\mathscr{B}$. $\mathrm{Si}$, pour tout $r \in\left(\boldsymbol{R}_{+}^{*}\right)^{I},\left(f_{j}\right)_{j \in J}$ est une $r$-famille (resp.: $r$-base) de décomposition de $E$ associée à $\mathscr{B}$, on dit que $\left(f_{j}\right)_{j \in J}$ est une famille (resp.: base) de décomposition de $E$ associée à $\mathscr{B}$. $\mathrm{Si}$, pour tout $r \in\left(\boldsymbol{R}_{+}^{*}\right)^{I},\left(f_{j}\right)_{j \in J}$ est une $r$-famille (resp.: $r$-base) de décomposition forte de $E$ associée à $\mathscr{B}$, on dit que $\left(f_{j}\right)_{j \in J}$ est une famille (resp.: base) de décomposition forte de $E$ associée à $\mathscr{B}$. Remarquons que si $\left(f_{j}\right)_{j \in J}$ est une $r$-famille de décomposition forte ou non associée à $\mathscr{B}$, alors $I$ est sans plus grand élément. 
2.1.2. ThÉorème 2.1 (voir [1], Théorème 3.1 et 3.1. bis ou [2], p. 900). Soient $E$ un espace vectoriel de dimension infinie, $\left(e_{i}\right)_{\imath \in I}$ une base bien ordonnée de $E, r$ un élément quelconque de $\left(\boldsymbol{R}_{+}^{*}\right)^{I}$ et $\left(f_{j}\right)_{j \in J}$ une r-famille de décomposition (resp.: décomposition forte) de $E$ associée à $\left(e_{i}\right)_{i \in I}$. On a alors:

$$
\begin{gathered}
C S\left[\bigcup_{i \in I}\left\{e_{i}\right\}\right]+C S\left[\bigcup_{j \in J}\left\{f_{j}\right\}\right]=E \\
\text { (resp.: } \left.C S\left[\bigcup_{i \in I}\left\{e_{i}\right\}\right]+C\left[\bigcup_{j \in J}\left\{f_{j}\right\}\right]=E\right) .
\end{gathered}
$$

2.1.3. Premier exemple (voir [1], paragraphe 3.3.1-ou [2], p. 900).

Soient $E$ un espace vectoriel de dimension infinie dénombrable, $\left(e_{n}\right)_{n \in N^{*}}$ une base de $E\left(N^{*}\right.$ est muni de l'ordre naturel).

Soit $(m(n))_{n \in N^{*}}$ une suite strictement croissante d'entiers avec $m(1)>0$. Posons, pour tout $n \in N^{*}$,

$$
f_{n}=a_{n, 1} e_{1}+a_{n, 2} e_{2}+\cdots+a_{n, m(n)} e_{m(n)},
$$

les $a_{n, i}$ vérifiant les propriétés $P_{1}(n), P_{2}(n), P_{3}(n)$ suivantes:

$P_{1}(n):\left|a_{n, i}\right| \geqq n$ pour $1 \leqq i \leqq m(n-1)$.

$P_{2}(n)$ : Il existe $i$ avec $m(n-1)<i \leqq m(n)$ tel que $a_{n, i} \neq 0$.

$$
\begin{aligned}
P_{3}(n) & : \frac{\left|a_{n, i}\right|}{\left|a_{n, i+1}\right|+\left|a_{n, i+2}\right|+\cdots+\left|a_{n, m(n)}\right|} \\
& \geqq n \text { pour } 1 \leqq i \leqq m(n-1) .
\end{aligned}
$$

On peut vérifier que $\left(f_{n}\right)_{n \in N^{*}}$ est une famille libre de décomposition de $E$ associée à $\left(e_{n}\right)_{n \in N^{*}}$.

Si de plus, les $a_{n, i}$ vérifient les propriétés $P_{4}(n)$ suivantes:

$P_{4}(n): a_{n, i}$ est $d u$ signe de $(-1)^{n+i}$ pour $1 \leqq i \leqq m(n-1)$,

$\left(f_{n}\right)_{n \in N^{*}}$ est alors une famille libre de décomposition forte de $E$ associée à $\left(e_{n}\right)_{n \in N^{*}}$.

2.1.4. SeCOND EXemple (voir [1], paragraphes 3.3.2 et 5.1.2).

Soient $E$ un espace vectoriel de dimension infinie dénombrable, $\left(e_{n}\right)_{n \in N^{*}}$ une base de $E$. Soit $\Phi^{\prime}$ l'ensemble des applications $\varphi$ de $N^{*}$ dans $\boldsymbol{R}_{+}^{*}$ telles que:

$$
\lim _{n \rightarrow+\infty} \frac{\varphi(n)}{\sum_{k=1}^{n-1} \varphi(k)}=+\infty .
$$

Soit $\phi \in \Phi^{\prime}$. Posons, pour tout $n \in N^{*}$, 


$$
f_{n}=\sum_{p=1}^{n}(-1)^{n+1-p} \varphi(n+1-p) e_{p} .
$$

$\left(f_{n}\right)_{n \in N^{*}}$ est une base de décomposition forte de $E$ associée $\grave{a}\left(e_{n}\right)_{n \in N^{*}}$.

2.1.5. Le théorème suivant donne un résultat dans le cas où $I$ est un ensemble produit et permet ainsi de construire des familles de décomposition dans tout espace vectoriel de dimension infinie.

THÉorème 2.2 (voir [1], Théorème 3.2-ou [2], p. 901). Soient $S$ et $T$ deux ensembles bien ordonnés, $S$ étant non vide et $T$ étant infini; soit $I=S \times T$ supposé bien ordonné par l'ordre lexicographique. Soit $E$ un espace vectoriel somme directe $\bigoplus_{\mathrm{s} \in S} E_{\mathrm{s}}$ où, pour tout $s \in S, E_{s}$ est un espace vectoriel ayant pour base $\left(e_{t}^{s}\right)_{t \in T}$. Considérons, pour tout $s \in S$, un élément donné $r^{s}=\left(r_{t}^{s}\right)_{t \in T}$ de $\left(\boldsymbol{R}_{+}^{*}\right)^{T}$ et désignons par $r=\left(r_{i}\right)_{i \in I}$ l'élément de $\left(\boldsymbol{R}_{+}^{*}\right)^{I}$ tel que, pour tout $i=(s, t) \in I$, on ait $r_{i}=r_{t}^{s}$.

Si, pour tout $s \in S$, la famille $\left(f_{u}^{s}\right)_{u \in U}$ de vecteurs de $r_{s}$ est une $r^{s}$-famille (resp.: $r^{s}$-base) de décomposition (forte) de $E_{s}$ associée à la base $\left(e_{t}^{s}\right)_{t \in T}$, alors la famille $\left(f_{u}^{s}\right)_{(s, u) \in S \times U}$ est une r-famille (resp.: $r$-base) de décomposition (forte) de $E$ associée à la base $\left(e_{t}^{s}\right)_{(s, t) \in I}$.

2.2. Notion de convexe ubiquitaire-élémentaire et de convexe ubiquitaire-basique.

2.2.1. Base triangulatre (voir [1], Définition 5.1-ou [3], p. 1168). Soient $E$ un espace vectoriel, $\left(e_{i}\right)_{i \in I}$ et $\left(f_{i}\right)_{i \in I}$ deux bases bien ordonnées de $E$. On dit que $\left(f_{i}\right)_{i \in I}$ est une base triangulaire de $E$ relativement à la base $\left(e_{i}\right)_{i \in I}$ si, pour tout $j \in I, f_{j}=\sum_{i \in I} a_{j, i} e_{i}$ avec

$$
\left\{\begin{array}{l}
a_{j, i}=0 \text { pour tout } i>j \\
a_{j, j} \neq 0
\end{array}\right.
$$

2.2.2. DÉfinitions (voir [1], Définitions 5.2 et 5.3-ou [3], p. 1167). Soient $E$ un espace vectoriel de dimension infinie, $\left(e_{i}\right)_{i \in I}$ une base bien ordonnée de $E$ (sans plus grand élément), $\left(f_{j}\right)_{j \in J}$ une famille de décomposition forte de $E$ associée à $\left(e_{i}\right)_{i \in i}$ telle que, pour tout $j \in J$ avec $f_{j} \neq 0$, la dernière composante non nulle de $f_{j}$ dans la base $\left(e_{i}\right)_{i \in I}$ soit $<0$. Posons:

$$
\Gamma_{1}=C\left[\left(\bigcup_{i \in I}\left\{-e_{i}\right\}\right) \cup\left(\bigcup_{j \in J}\left\{f_{j}\right\}\right)\right] .
$$

Soit $\left(c_{i}\right)_{i \in I}$ une famille de points de $\Gamma_{1}$ tous distincts de 0 tels que, pour tout $i \in I$, la dernière composante non nulle de $c_{i}$ dans la base $\left(e_{i}\right)_{i \in I}$ ait un indice $>i$. 
Posons

$$
\left.\left.\Gamma_{2}=C\left[\Gamma_{1} \cup\left(\bigcup_{i \in I}\left\{e_{i}+t c_{i} \mid t \in\right] 0,1\right]\right\}\right)\right] .
$$

Tout convexe du type $\Gamma_{1}$ est appelé convexe élémentaire et tout convexe du type $\Gamma_{2}$ est appelé convexe ubiquitaire-élémentaire.

$\mathrm{Si}$, en outre, $\left(f_{j}\right)_{j \in J}$ est une base triangulaire de $E$ relativement à la base $\left(e_{i}\right)_{i \in I}$ (on suppose $J=I$ ), $\Gamma_{1}$ est appelé convexe basique et $\Gamma_{2}$ est appelé convexe ubiquitaire-basique.

Les définitions qui précèdent sont justifiées par le théorème suivant:

THÉorème 2.3 (voir [1], Théorème 5.1 -ou [3], p. 1167). Tout convexe ubiquitaire-élémentaire (donc tout convexe ubiquitaire-basique) est proprement ubiquitaire.

2.2.3. Rappelons aussi quelques résultats qui seront utiles dans les démonstrations de cet article.

LEMme 2.1 (voir [1], Lemme 5.1-ou [3], p. 1168). Soit, dans un espace vectoriel $E$, un convexe ubiquitaire $C$ contenant l'origine. Si $y \in E$, il existe $x \in C$ tel que, pour tout $t \in] 0,1], y+t x \in C$.

LeMme 2.2 (voir [1], Lemme 5.5). Soient, dans un espace vectoriel $E, n$ convexes $A_{1}, A_{2}, \cdots, A_{n}$ tels que $C\left[\bigcup_{i=1}^{n} A_{i}\right]=E$, et $A_{1}^{\prime}, A_{2}^{\prime}$, $\cdots, A_{n}^{\prime}$ des translatés respectifs de $A_{1}, A_{2}, \cdots, A_{n}$ dans des translations $t_{1}, t_{2}, \cdots, t_{n}$. Alors, on a $C\left[\bigcup_{i=1}^{n} A_{i}^{\prime}\right]=E$.

ThÉoRème 2.4 (voir [1], Lemme 5.4 et Théorème 5.2). Soit, dans un espace vectoriel $E$ de dimension infinie dénombrable, un convexe ubiquitaire $U$ contenant l'origine. Alors $U$ contient un convexe ubiquitaire-basique qui est associé à une base de $E$ indexée par $N^{*}$ muni de son bon ordre naturel.

2.3. Notion de cône d'infinitude.

2.3.1. Définition (voir [1], Définition 1.1). Soit, dans un espace vectoriel $E$, une partie $A$; le cône d'infinitude de $A$, noté $\mathscr{T}[A]$, est le cône pointé de sommet 0 qui est réunion des demi-droites issues de 0 dont une translatée au moins est contenue dans $A$.

2.3.2. Rappelons quelques propriétés indispensables dans la suite.

Proposition 2.1 (voir [1], Proposition 1.7). Une partie $A$ convexe d'un espace vectoriel $E$ est linéairement bornée si et seulement si 
$\mathscr{T}[A]=\{0\}$.

ThEorìme 2.5 (voir [1], Corollaire 2.3). Soient, dans un espace vectoriel $E$, deux convexes $A$ et $B$ linéairement bornés dont l'un est de dimension finie. Alors $A+B$ et $C[A \cup B]$ sont linéairement bornés.

REmarque. Notons que dans le théorème précédent la condition de dimension finie est essentielle ainsi que le montre le procédé de construction de convexes $A$ et $B$ linéairement bornés et tels que $A+$ $B$ et $C[A \cup B]$ soient non linéairement bornés (voir [1], page 17).

Lemme 2.3 (voir [1], Lemme 2.5). Soient, dans un espace vectoriel $E, n$ parties $A_{1}, A_{2}, \cdots, A_{n}$ et $n$ points $x_{1}, x_{2}, \cdots, x_{n}$. Posons, pour $1 \leqq i \leqq n, A_{i}^{\prime}=A_{i}-\left\{x_{i}\right\}$. Alors, on $a$ :

$$
\mathscr{T}\left[C\left[\bigcup_{i=1}^{n} A_{i}^{\prime}\right]\right]=\mathscr{T}\left[C\left[\bigcup_{i=1}^{n} A_{i}\right]\right] \text {. }
$$

ThEorème 2.6 (voir [1], Théorème 2.3). Soient, dans un espace vectoriel $E$, une somme directe $F=\bigoplus_{i \in I} E_{i}$ de sous-espaces vectoriels $E_{i}$ de $E$ et, pour tout $i \in I$, un convexe $A_{i}$ de $E_{i}$. Alors, on a:

$$
\mathscr{T}\left[C\left[\bigcup_{i \in I} A_{i}\right]\right]=C\left[\bigcup_{i \in I} \mathscr{T}\left[A_{i}\right]\right]
$$

III. Deux conditions suffisantes permettant d'affirmer qu'un convexe ubiquitaire-elementaire (resp.: ubiquitaire-basique) est lineairement borne. Ces conditions (Théorèmes 3.1 et 3.2) reposent sur les Lemmes 3.1 et 3.2.

3.1. Définition 3.1. Soient $E$ un espace vectoriel non nul, $\mathscr{B}=\left(e_{i}\right)_{i \in I}$ une base totalement ordonnée de $E$ et $\left(g_{j}\right)_{j \in J}$ une famille de vecteurs de $E$. Désignons, pour tout $i \in I$, par $J_{i}$ l'ensemble des $j \in J$ pour lesquels $g_{j}$ a une dernière composante non nulle dans $\mathscr{B}$ d'indice $i$.

$\mathrm{Si}$, pour tout $i \in I, J_{i}$ est fini, on dira que $\left(g_{j}\right)_{j \in J}$ possède la propriété $\mathscr{P}$ relativement à $\mathscr{B}$.

3.2. Lemme 3.1. Soit $A$ une partie (resp.: une partie convexe) d'un espace vectoriel $E$. $A$ ne contient pas de demi-droite (resp.: A est linéairement bornée) si et seulement s'il existe une famille $\left(E_{j}\right)_{j \in J}$ filtrante croissante de sous-espaces vectoriels de $E$ telle que $E=\bigcup_{j \in J} E_{j}$ et telle que, pour tout $j \in J, A \cap E_{j}$ ne contienne pas de demi-droite (resp.: $A \cap E_{j}$ soit linéairement bornée). 
Démonstration. Supposons d'abord que $A$ soit une partie quelconque. La condition nécessaire est évidente: Il suffit de prendre pour famille $\left(E_{j}\right)_{j \in J}$ la famille réduite au seul élément $E$.

Montrons que la condition est suffisante. Soit $\Delta$ une demi-droite; il existe $j \in J$ tel que $\Delta \subset E_{j}$. Si $\Delta$ était contenue dans $A$, on aurait $\Delta \subset A \cap E_{j}$.

Le cas où $A$ est un convexe est une conséquence du cas précédent et du fait qu'un convexe est linéairement borné si et seulement s'il ne contient aucune demi-droite.

3.3. LEMmE 3.2. Soit $\mathscr{B}=\left(e_{i}\right)_{i \in I}$ une base bien ordonnée de l'espace vectoriel $E$ et soit $\left(C_{i}\right)_{i \in I}$ une famille de convexes de $E$ possédant les propriétés $\left(h_{1}\right)$ et $\left(h_{2}\right)$ :

$\left(h_{1}\right)$ : Pour tout $i \in I$, les points non nuls de $C_{i}$ ont une dernière composante non nulle dans $\mathscr{B}$ qui est $<0$ et qui a pour indice $i$.

$\left(h_{2}\right)$ : Pour tout $i \in I, C_{i}$ est linéairement borné et de dimension finie.

Alors le convexe $C=C\left[\bigcup_{i \in I} C_{i}\right]$ est linéairement borné.

Démonstration. D'après le Théorème 2.5, $C$ (resp.: $C_{i}$ ) est linéairement borné si et seulement si $C[C \cup\{0\}]$ (resp.: $C\left[C_{i} \cup\{0\}\right]$ ) l'est aussi; on peut donc supposer dans la suite que chaque $C_{i}$ contient 0 .

Posons, pour tout $l \in I, E_{l}=S\left[\bigcup_{i \leqq l}\left\{e_{i}\right\}\right], \Gamma_{l}=C\left[\bigcup_{i \leqq l} C_{i}\right]$.

D'après $\left(h_{1}\right)$, il est clair qu'on a:

$$
\forall l \in I, \Gamma_{l}=C \cap E_{l} .
$$

Montrons maintenant par récurrence transfinie sur $I$ que, pour tout $l \in I, \Gamma_{l}$ est linéairement borné, ce qui assurera d'après le Lemme 3.1 que $C$ est linéairement borné.

La propriété est vraie pour le plus petit élément $i_{0}$ de $I$ car $\Gamma_{i_{0}}=$ $C_{i_{0}}$. Soit $l \in I$; supposons la propriété vraie pour tous les $i \in I$ tels que $i<l$, et montrons qu'elle est vraie pour $l$.

Posons $D_{l}=C\left[\bigcup_{i<l} C_{i}\right]$.

La propriété $\left(h_{1}\right)$ montre qu'on a:

$$
\forall_{i}<l, D_{l} \cap E_{i}=\Gamma_{i} .
$$

D'après l'hypothèse de récurrence, pour tout $i<l, \Gamma_{i}$ est linéairement borné; il en résulte, d'après (2), l'inclusion $D_{l} \subset \bigcup_{i<l} E_{i}$ et le Lemme 3.1, que $D_{l}$ est linéairement borné.

Puisque $\Gamma_{l}=C\left[D_{l} \cup C_{l}\right]$, il résulte de $\left(h_{2}\right)$ et du Théorème 2.5 que $\Gamma_{l}$ est linéairement borné.

3.4. ThÉoRÈme 3.1. Soit $\mathscr{B}=\left(e_{i}\right)_{i \in I}$ une base bien ordonnée 
de l'espace vectoriel $E$; soit $\left(g_{j}\right)_{j \in J}$ (resp.: $\left.\left(f_{j}\right)_{j \in J}\right)$ une famille de vecteurs de $E$ (resp.: une famille de décomposition forte de $E$ associée à $\mathscr{B}$ ) telle que, pour tout $j \in J$ avec $g_{j} \neq 0$ (resp.: $f_{j} \neq 0$ ), la dernière composante non nulle de $g_{j}$ (resp.: $f_{j}$ ) dans $\mathscr{B}$ soit $<0$. Posons

$$
\Gamma_{1}=C\left[\left(\bigcup_{i \in I}\left\{-e_{i}\right\}\right) \cup\left(\bigcup_{j \in J}\left\{f_{j}\right\}\right)\right] .
$$

Soit $\left(c_{i}\right)_{i \in I}$ une famille de points de $\Gamma_{1}$ tous distincts de 0 telle que, pour tout $i \in I$, la dernière composante non nulle de $c_{i}$ dans $\mathscr{B}$ ait $u$ indice $>i$. Posons

$$
\left.\left.\Gamma_{2}=C\left[\Gamma_{1} \cup\left(\bigcup_{i \in I}\left\{e_{i}+t c_{i} \mid t \in\right] 0,1\right]\right\}\right)\right] .
$$

(a) Si $\left(g_{j}\right)_{j \in J}$ possède la propriété $\mathscr{P}$ relativement à $\mathscr{B}$, alors le convexe $C\left[\left(\bigcup_{i \in I}\left\{-e_{i}\right\}\right) \cup\left(\bigcup_{j \in J}\left\{g_{j}\right\}\right)\right]$ est linéairement borné.

(b) Si $\left(f_{j}\right)_{j \in J}$ possède la propriété $\mathscr{P}$ relativement à $\mathscr{B}$, le convexe élémentaire $\Gamma_{1}$ est linéairement borné. Si, de plus, $\left(c_{i}\right)_{i \in I}$ possède la propriété $\mathscr{P}$ relativement à $\mathscr{B}$, alors le convexe ubiquitaire-élémentaire $\Gamma_{2}$ est aussi linéairement borné.

(c) Tout convexe basique est linéairement borné.

(d) $S i\left(f_{j}\right)_{j \in J}$ est une base triangulaire de $E$ relativement à $\mathscr{B}$ et si $\left(c_{i}\right)_{i \in I}$ possède la propriété $\mathscr{P}$ relativement à $\mathscr{B}$, le convexe ubiquitaire-basique $\Gamma_{2}$ est linéairement borné.

Démonstration. Démontrons l'assertion (b); les autres assertions se démontrent d'une manière analogue (une base triangulaire de $E$ relativement à $\mathscr{B}$ possède évidemment la propriété $\mathscr{P}$ relativement à $\mathscr{B})$.

Désignons, pour tout $i \in I$, par $J_{i}$ (resp.: $I_{i}$ ) le sous-ensemble de $J$ (resp.: $I$ ) constitué par les indices $j \in J$ (resp.: $j \in I$ ) pour lesquels $f_{j}\left(\right.$ resp.: $\left.c_{j}\right)$ ait une dernière composante non nulle dans $\mathscr{B}$ d'indice $i$.

Il suffit de poser, pour tout $i \in I$,

$$
\left.\left.C_{i}=C\left[\left\{-e_{i}\right\} \cup\left(\bigcup_{j \in J_{i}}\left\{f_{j}\right\}\right) \cup\left(\bigcup_{j \in I_{i}}\left\{e_{j}+t c_{j} \mid t \in\right] 0,1\right]\right\}\right)\right]
$$

et d'appliquer le Lemme 3.2 et le Théorème 2.5 .

3.5. Cas particulier de la dimension infinie dénombrable.

ThÉonème 3.2. Avec les notations du Théorème 3.1, si E est de dimension infinie dénombrable et si $I=N^{*}$, où $N^{*}$ est muni d'un bon ordre isomorphe au bon ordre naturel, on $a$ :

(1) Les assertions $a$ et $b$ restent vraies lorsqu'on remplace 
l'hypothèse " $\left(g_{j}\right)_{j \in J}\left(\right.$ resp.: $\left.\left(f_{j}\right)_{j \in J}\right)$ possède la propriété $\mathscr{P}$ relativement à $\mathscr{B} "$ par l'hypothèse " $\left(g_{j}\right)_{j \in J}$ (resp.: $\left.\left(f_{j}\right)_{j \in J}\right)$ est libre".

Les assertions $b$ et d restent vraies lorsqu'on supprime l'hypothèse " $\left(c_{i}\right)_{i \in I}$ possède la propriété $\mathscr{P}$ relativement à $\mathscr{B} "$.

(2) Soit $\left(K_{p}\right)_{p \in P}$ une famille finie non vide de convexes linéairement bornés de $E$ tels que, pour tout $p \in P$, les points non nuls de $K_{p}$ aient une dernière composante non nulle dans $\mathscr{B}$ qui est $<0$. Alors le convexe $C=C\left[\bigcup_{p \in P} K_{p}\right]$ est linéairement borné.

Démonstration. (1) Cette partie résulte du fait que, lorsque $N^{*}$ est muni d'un bon ordre isomorphe au bon ordre naturel, la famille $\left(c_{i}\right)_{i \in I}$ vérifie la propriété $\mathscr{P}$ relativement à $\mathscr{B}$, et la famille $\left(g_{j}\right)_{j \in J}$ vérifie $\mathscr{P}$ relativement à $\mathscr{B}$ si elle est libre.

(2) Désignons, pour tout $n \in N^{*}$, par $F_{n}$ l'ensemble des vecteurs de $E$ formé par le vecteur 0 et par les vecteurs non nuls dont la dernière composante non nulle est $<0$ et a pour indice $n$.

Il suffit de poser, pour tout $n \in N^{*}, C_{n}=C\left[\bigcup_{p \in P}\left(K_{p} \cap F_{n}\right)\right]$ et d'appliquer le Lemme 3.2.

IV. Remarques. Les remarques qui suivent montrent l'importance des hypothèses mises en jeu aux Théorèmes 3.1 et 3.2 et au Lemme 3.2 .

4.1. L'exemple suivant montre l'importance de l'hypothèse de bon ordre faite sur I au Lemme 3.2 et au Théorème 3.1.

EXEMPLE. Soient $E$ un espace vectoriel de dimension infinie dénombrable, $\mathscr{B}=\left(e_{n}\right)_{n \in N}$ une base de $E$ supposée totalement ordonnée (mais non bien ordonnée) de la façon suivante: l'ordre sur $N^{*}$ est l'ordre inverse de l'ordre naturel et 0 est décrété $<$ à tout élément de $N^{*}$.

Posons $g_{0}=-e_{0}$ et, pour tout $n \in N^{*}, g_{n}=2 n e_{0}+e_{n+1}-e_{n}$. La famille $\left(g_{n}\right)_{n \in N}$ possède la propriété $\mathscr{P}$ relativement à $\mathscr{B}$ et est telle que, pour tout $n \in N$, la dernière composante non nulle de $g_{n}$ dans $\mathscr{B}$ est $<0$. Pourtant le convexe $C=C\left[\bigcup_{n \in N}\left\{-e_{n}, g_{n}\right\}\right]$ n'est pas linéairement borné. En effet, pour tout $n \in N^{*}$,

$$
\begin{aligned}
x_{n}= & \frac{1}{n}\left(g_{1}+g_{2}+\cdots+g_{n}\right)=(n+1) e_{0}+\frac{1}{n} e_{n+1}-\frac{1}{n} e_{1} \in C, \\
& \operatorname{donc} \frac{1}{2} x_{n}+\frac{1}{2}\left[\frac{1}{n}\left(-e_{n+1}\right)+\frac{n-1}{n}\left(-e_{1}\right)\right]=\frac{n+1}{2} e_{0}-\frac{1}{2} e_{1} \in C .
\end{aligned}
$$

Ainsi $C$ contient la demi-droite $\Delta=\left\{\lambda e_{0}-1 / 2 e_{1} \mid \lambda \geqq 1\right\}$. 
4.2. L'exemple suivant montre que le (a) du Théorème 3.1 est mis en défaut lorsque la famille $\left(g_{j}\right)_{j \in J}$ ne possède pas la propriété $\mathscr{P}$ relativement à $\mathscr{B}$.

Exemple. Soient $E$ un espace vectoriel de dimension infinie dénombrable, $\mathscr{B}=\left(e_{n}\right)_{n \in N^{*}}$ une base de $E$ supposée bien ordonnée de la façon suivante: tout entier pair est $<$ à tout entier impair, l'ensemble des entiers pairs et l'ensemble des entiers impairs étant ordonnés par l'ordre induit par l'ordre naturel sur $N^{*}$; on définit ainsi un bon ordre sur $N^{*}$.

Posons, pour tout $n \in N^{*}, g_{n}=e_{2 n}-n e_{1}$.

La famille $\left(g_{n}\right)_{n \in N^{*}}$ est telle que, pour tout $n \in N^{*}$, la dernière composante non nulle de $g_{n}$ dans $\mathscr{B}$ est $<0$, mais ne possède pas la propriété $\mathscr{P}$ relativement à $\mathscr{B}$.

On vérifie aisément que le convexe $C\left[\bigcup_{n \in N^{*}}\left\{-e_{n}, g_{n}\right\}\right]$ contient la demi-droite $\Delta=\left\{\lambda e_{1} \mid \lambda \leqq-1 / 2\right\}$.

4.3. L'exemple suivant montre que le (b) du Théorème 3.1 est mis en défaut lorsque la famille $\left(f_{j}\right)_{j \in J}$ ne possède pas la propriété $\mathscr{P}$ relativement à $\mathscr{B}$; ainsi il existe des convexes élémentaires non linéairement bornés.

Exemple. Soient $E$ un espace vectoriel de dimension infinie dénombrable, $\mathscr{B}=\left(e_{n}\right)_{n \in N^{*}}$ une base de $E$ supposée bien ordonnée comme à l'exemple précédent.

Soit $\phi \in \Phi^{\prime}$ (voir 2.1.4).

Posons, pour tout $n \in N^{*}, f_{4 n}=e_{2 n}-n e_{1}$, et, pour tout $n \in N$,

$$
\left\{\begin{array}{l}
f_{2 n+1}=\sum_{p=0}^{n}(-1)^{n+1-p} \varphi(n+1-p) e_{2 p+1} \\
f_{4 n+2}=\sum_{p=1}^{n}(-1)^{n+1-p} \varphi(n+1-p) e_{2 p} .
\end{array}\right.
$$

On vérifie aisément que la famille $\left(f_{n}\right)_{n \in N^{*}}$ est une famille de décomposition forte de $E$ associée à $\mathscr{B}$ qui est telle que, pour tout $n \in N^{*}$, la dernière composante non nulle de $f_{n}$ dans $\mathscr{B}$ est $<0$; elle ne possède pas la propriété $\mathscr{P}$ relativement à $\mathscr{B}$. On vérifie aisément que le convexe élémentaire $\Gamma_{1}=C\left[\bigcup_{n \in N^{*}}\left\{-e_{n}, f_{n}\right\}\right]$ contient la demidroite $\Delta=\left\{\lambda e_{1} \mid \lambda \leqq-1 / 2\right\}$.

4.4. L'exemple suivant montre que le (d) du Théorème 3.1 est mis en défaut lorsque la famille $\left(c_{i}\right)_{\imath \in I}$ ne possède pas la propriété $\mathscr{P}$ relativement à $\mathscr{B}$; ainsi, bien que tout convexe basique soit linéairement borné, il existe des convexes ubiquitaires-basiques non linéairement bornés (et ceci même en dimension infinie dénombrable). 
Exemple. Soient $E$ un espace vectoriel de dimension infinie dénombrable, $\mathscr{B}=\left(e_{n}\right)_{n \in N^{*}}$ une base de $E$ supposée bien ordonnée comme à l'exemple précédent.

Soit $\left(f_{2 n}\right)_{n \in N^{*}}\left(\right.$ resp.: $\left.\left(f_{2 n+1}\right)_{n \in N}\right)$ une base triangulaire de décomposition forte de $S_{2}=S\left[\bigcup_{n \in N^{*}}\left\{e_{2 n}\right\}\right]$ (resp.: $\left.S_{1}=S\left[\bigcup_{n \in N}\left\{e_{2 n+1}\right\}\right]\right)$ associée à la base $\left(e_{2 n}\right)_{n \in N^{*}}\left(\right.$ resp.: $\left.\left(e_{2 n+1}\right)_{n \in N}\right)$ de $S_{2}$ (resp.: $\left.S_{1}\right)$ telle que, pour tout $n \in$ $N^{*}($ resp.: $n \in N)$, la dernière composante non nulle de $f_{2 n}$ (resp.: $f_{2 n+1}$ ) dans $\mathscr{B}$ soit $<0$.

La famille $\left(f_{n}\right)_{n \in N^{*}}$ est une base triangulaire de décomposition forte de $E$ associée à $\mathscr{B}$ (voir Théorème 2.2) et le convexe $\Gamma_{1}=$ $C\left[\bigcup_{n \in N^{*}}\left\{-e_{n}, f_{n}\right\}\right]$ est un convexe basique.

Soit $\left(c_{n}\right)_{n \in N^{*}}$ une famille de points de $\Gamma_{1}$ telle que, pour tout $n \in$ $N^{*}$, la dernière composante non nulle de $c_{n}$ dans $\mathscr{B}$ ait un indice $>n$ et telle que, pour tout $n \in N^{*}, c_{2 n}=-e_{1}$. Puisque $2 n<1$, il est clair que la famille $\left(c_{n}\right)_{n \in N^{*}}$ ne possède pas la propriété $\mathscr{P}$ relativement à $\mathscr{B}$.

Montrons que le convexe ubiquitaire-basique $\Gamma_{2}$ avec

$$
\left.\left.\Gamma_{2}=C\left[\Gamma_{1} \cup\left(\bigcup_{n \in \mathbb{N}^{*}}\left\{e_{n}+t c_{n} \mid t \varepsilon\right] 0,1\right]\right\}\right)\right]
$$

n'est pas linéairement borné.

$\Gamma_{2}$ contient $A=C\left[\bigcup_{n \in N^{*}}\left\{-e_{2 n}\right\}\right], B=C\left[\bigcup_{n \in N^{*}}\left\{e_{2 n}-e_{1}\right\}\right]$ et $C=$ $C\left[\bigcup_{n \in N^{*}}\left\{f_{2 n}\right\}\right]$. Ainsi le cône d'infinitude de $\Gamma_{2}$ contient le cône d' infinitude de $C[A \cup B \cup C]$. Puisque $-A$ est un translaté de $B$, le cône d'infinitude de $C[A \cup B \cup C]$ coincide avec celui de $C[A \cup(-A) \cup C]$ (voir Lemme 2.3) qui n'est autre que $S_{2}$ tout entier car $C[A \cup(-A) \cup$ $C]=S_{2}$ (voir Théorème 2.1). Ainsi, $\Gamma_{2}$ n'est pas linéairement borné car son cône d'infinitude n'est pas réduit à $\{0\}$ (voir Proposition 2.1).

4.5. Lorsque le bon ordre sur $N^{*}$ n'est pas isomorphe au bon ordre naturel, l'exemple du 4.2 montre que la partie 2 du Théorème 3.2 est mise en défaut: En effet les convexes $C\left[\bigcup_{n \in N^{*}}\left\{-e_{n}\right\}\right]$ et $C\left[\bigcup_{n \in N^{*}}\left\{g_{n}\right\}\right]$ sont linéairement bornés car les familles $\left(-e_{n}\right)_{n \in N^{*}}$ et $\left(g_{n}\right)_{n \in N^{*}}$ sont libres (voir Théorème 2.6 et Proposition 2.1). Lorsque le bon ordre sur $N^{*}$ n'est pas isomorphe au bon ordre naturel, l'exemple du 4.3 (resp.: 4.4) montre qu'une famille libre de décomposition forte $\left(f_{j}\right)_{j \in J}$ (resp.: une famille $\left.\left(c_{i}\right)_{i \in N^{*}}\right)$ ne vérifie pas nécessairement la propriété $\mathscr{P}$ relativement à $\mathscr{B}=\left(e_{n}\right)_{n \in N^{*}}$ et montre aussi que, même en dimension infinie dénombrable, un convexe élémentaire (resp.: un convexe ubiquitaire-basique) peut être non linéairement borné.

V. Convexes ubiquitaires et convexes ubiquitaires lineairement bornes de dimension infinie denombrable. 
5.1. LEMmE 5.1. Soient $E$ un espace vectoriel de dimension infinite, $\mathscr{B}=\left(e_{i}\right)_{i \in I}$ une base bien ordonnée de $E,\left(f_{j}\right)_{j \in J}$ une famille de décomposition forte de $E$ associée à $\mathscr{B}$. Posons:

$$
A=C\left[\bigcup_{i \in I}\left\{e_{i}\right\}\right] \text { et } B=C\left[\left(\bigcup_{i \in I}\left\{-e_{i}\right\}\right) \cup\left(\bigcup_{j \in J}\left\{f_{j}\right\}\right)\right] \text {. }
$$

On a alors $2 B+A=E$.

Démonstration. Soit $k$ un indice fixé dans $I$. Posons $B^{\prime}=B+$ $\left\{e_{k}\right\}$ et $A^{\prime}=A-\left\{e_{k}\right\}$. On $a$ :

$$
2 B+A+\left\{e_{k}\right\}=2 B^{\prime}+A^{\prime} .
$$

Montrons que $C[B \cup A]=E$.

En effet $C[B \cup A]$ contient $C S\left[\bigcup_{i \in I}\left\{e_{i}\right\}\right]$ et $C\left[\bigcup_{j \in J}\left\{f_{j}\right\}\right]$. Ainsi, on $a$ :

$$
C[B \cup A] \supset \frac{1}{2}\left(C S\left[\bigcup_{i \in I}\left\{e_{i}\right\}\right]+C\left[\bigcup_{j \in J}\left\{f_{j}\right\}\right]\right),
$$

et le Théorème 2.1 montre que $C[B \cup A]=E$.

On en déduit, d'après le Lemme 2.2 que $C\left[B^{\prime} \cup A^{\prime}\right]=E$. Puisque $0 \in B^{\prime} \cap A^{\prime}$, il en résulte que $C\left[2 B^{\prime} \cup A^{\prime}\right]=E$, donc que $2 B^{\prime}+A^{\prime}=E$. D'après (1), on $a$ alors $2 B+A=E$.

5.2. LEMME 5.2. Soient $E$ un espace vectoriel et $U$ un convexe ubiquitaire dans $E$. Supposons qu'il existe une famille non vide $\left(F_{j}\right)_{j \in J}$ de sous-espaces vectoriels de dimension infinie dénombrable de $E$ telle que la somme $\sum_{j \in J} F_{j}$ soit directe et de codimension dénombrable dans $E$ et telle que, pour tout $j \in J, U \cap F_{j}$ soit ubiquitaire dans $F_{j}$. Alors, il existe une famille $\left(E_{i}\right)_{i \in I}$ de sous-espaces vectoriels de dimension infinie dénombrable de $E$ telle que la somme $\sum_{i \in I} E_{i}$ soit directe et égale à $E$ et telle que, pour tout $i \in I, U \cap E_{i}$ soit ubiquitaire dans $E_{i}$.

Démonstration. Si $\bigoplus_{j \in J} F_{j}=E$, c'est terminé. Supposons donc que $\bigoplus_{j \in J} F_{j} \neq E$. Soit $S$ un sous-espace vectoriel supplémentaire de $\bigoplus_{j \in J} F_{j}$ dans $E$ et soit $\left(e_{l}\right)_{l \in L}$ une base de $S$ ( $L$ est dénombrable).

Soit $j_{0}$ un indice quelconque dans $J$. Posons:

$$
\left\{\begin{array}{l}
G_{j_{0}}=F_{j_{0}} \oplus S \\
G_{j}=F_{j} \text { pour tout } j \in J \sim\left\{j_{0}\right\} .
\end{array}\right.
$$

Puisque $U$ est ubiquitaire dans $E$, pour tout $(l, n) \in L \times Z$, il existe $x_{(l, n)} \in U$ tel que l'on ait (1):

$$
\left\{\lambda n e_{l}+(1-\lambda) x_{(l, n)} \mid \lambda \in[0,1[\} \subset U .\right.
$$


Désignons, pour tout $(l, n) \in L \times Z$, par $K(l, n)$ le sous-ensemble fini de $J$ constitué par les indices $j$ tels que $x_{(l, n)}$ ait une composante nonulle suivant $G_{j}$ (remarquons que $E=\bigoplus_{j \in J} G_{j}$ ). Posons

$$
K=\left\{j_{0}\right\} \cup\left(\bigcup_{(l, n) \in L \times Z} K(l, n)\right) .
$$

$K$ est un sous-ensemble dénombrable de $J$ et pour tout $(l, n) \in L \times Z$, $x_{(l, n)} \in U \cap \bigoplus_{j \in K} G_{j}$. Posons $I=\left\{j_{0}\right\} \cup(J \sim K), E_{j_{0}}=\bigoplus_{j \in K} G_{j}$ et, pour tout $j \in J \sim K, E_{j}=G_{j}$. La famille $\left(E_{i}\right)_{i \in I}$ vérifie les conclusions du lemme: En effet, les $E_{i}$ sont tous de dimension infinie dénombrable; leur somme est directe et égale à $E$ (car $E=\bigoplus_{j \in J} G_{j}$ ). En outre, pour tout $j \in J \sim K, \operatorname{lin}\left(U \cap E_{j}\right)=\operatorname{lin}\left(U \cap F_{j}\right)=F_{j}=E_{j}$ (par hypothèse); d'autre part, par construction de $E_{j_{0}}$ et, compte tenu de (1), $U \cap E_{j_{0}}$ contient les intervalles $\left.] n e_{l}, x_{(l, n)}\right]$, donc $\operatorname{lin}\left(U \cap E_{j_{0}}\right)$ contient les $n e_{l}$ donc aussi $S$. De plus, par construction de $E_{j_{0}}$, lin $\left(U \cap E_{j_{0}}\right)$ contient, pour tout $j \in K$, lin $\left(U \cap F_{j}\right)$ donc aussi $F_{j}$ (par hypothèse); par suite, $\operatorname{lin}\left(U \cap E_{j_{0}}\right)=E_{j_{0}}$.

5.3. ThÉов̀ेme 5.1. Soit, dans un espace vectoriel $E$ de dimension infinie, un convexe ubiquitaire $U$ contenant l'origine. Alors il existe une famille $\left(E_{j}\right)_{j \in J}$ de sous-espaces vectoriels de E telle que l'on ait (i), (ii), (iii) avec:

(i) Pour tout $j \in J, E_{j}$ est de dimension infinie dénombrable.

(ii) La somme des $E_{j}$ est directe et égale à $E$.

(iii) Pour tout $j \in J, U \cap E_{j}$ est ubiquitaire dans $E_{j}$.

Démonstration. Soit $\left(g_{i}\right)_{i \in I}$ une base bien ordonnée de $E$ telle que, pour tout $i \in I, g_{i} \in U$ (ceci est possible car $S[U]=E$ ).

Considérons, sur l'indice $l \in I$, la propriété récurrente $P(l)$ suivante: Il existe un sous-espace vectoriel $E_{l}$ de $E$ tel que l'on ait $\left(h_{1}\right)$ et $\left(h_{2}\right)$ avec:

$\left(h_{1}\right)$ : ou bien $E_{l}=\{0\}$.

ou bien $E_{l}$ est de dimension infinie dénombrable et $U \cap E_{l}$ est ubiquitaire dans $E_{l}$.

$\left(h_{2}\right)$ : la somme $\sum_{i \leqq l} E_{i}$ est directe et contient $g_{l}$.

Pour tout $l \in I$ tel que, pour tout $i<l(i \in I), P(i)$ soit vérifiée, on pose $F_{l}=\bigoplus_{i<l} E_{i}$.

Deux cas exhaustifs peuvent se présenter:

1er cas. Il existe $l \in I$ tel que l'on ait $P(i)$ pour tout $i<l(i \in I)$ et tel que $F_{l}$ soit de codimension finie.

L'ensemble des $i<l$ tels que $E_{i} \neq\{0\}$ est non vide puisque $E$ est de dimension infinie; le Lemme 5.2 montre alors que le Théorème 5.1 est vrai. 
$2^{\mathrm{e}}$ cas. Pour tout $l \in I$, si pour tout $i<l$, on a $P(i)$, alors $F_{l}$ est de codimension infinie.

Montrons alors par récurrence transfinie que $P(l)$ est vraie pour tout $l \in I$. Soit $l \in I$; supposons $P(i)$ vraie pour tout $i<l$ et montrons que $P(l)$ est vraie.

Si $g_{l} \in F_{l}$, on choisit $E_{l}=\{0\}$ et alors $P(l)$ est vraie. Supposons que $g_{l} \notin F_{l}$.

Construisons, par récurrence sur l'entier $n$, une suite libre $\left(e_{n}\right)_{n \in N^{*}}$ de vecteurs de $E$ et une suite libre $\left(f_{n}\right)_{n \in N^{*}}$ de vecteurs de $E$ satisfaisant aux propriétés $R_{1}(n), R_{2}(n), R_{3}(n), R_{4}(n), R_{5}(n)$ suivantes:

$$
\begin{aligned}
& R_{1}(n):-e_{n} \in U . \\
& R_{2}(n): e_{n} \notin F_{l} \oplus S\left[\left\{e_{1}, e_{2}, \cdots, e_{n-1}\right\}\right] . \\
& R_{3}(n): f_{n} \in U . \\
& R_{4}(n): f_{n}=a_{n, 1} e_{1}+a_{n, 2} e_{2}+\cdots+a_{n, n} e_{n},
\end{aligned}
$$

les $a_{n, i}$ vérifiant les propriétés $P_{1}(n), P_{2}(n), P_{3}(n), P_{4}(n)$ suivantes:

$$
\left\{\begin{array}{l}
P_{1}(n):\left|a_{n, i}\right| \geqq n \quad \text { pour } 1 \leqq i \leqq n-1 \\
P_{2}(n): a_{n, n}=-1 \cdot \\
P_{3}(n): \frac{\left|a_{n, i}\right|}{\left|a_{n, i+1}\right|+\left|a_{n, i+2}\right|+\cdots+\left|a_{n, n}\right|} \geqq n \quad \text { pour } \quad 1 \leqq i \leqq n-1 \\
P_{4}(n): a_{n, i} \text { est du signe de }(-1)^{n+i} \text { pour } 1 \leqq i \leqq n-1
\end{array}\right.
$$

$R_{5}(n): e_{n-1}-t e_{n} \in U$ pour tout $\left.\left.t \in\right] 0,1\right](n>1)$.

On pose $f_{1}=-e_{1}=g_{l}$.

Supposons $e_{1}, e_{2}, \cdots, e_{n}, f_{1}, f_{2}, \cdots, f_{n}$ construits.

Considérons un vecteur $w=\sum_{i=1}^{n} b_{i} e_{i}$, les coefficients $b_{i}$ vérifiant les propriétés $P_{3}^{\prime}(n)$ et $P_{4}^{\prime}(n)$ suivantes:

$$
\begin{aligned}
& P_{3}^{\prime}(n): \frac{\left|b_{n}\right|}{2} \geqq n+1, \frac{\left|b_{n-1}\right|}{2+\left|b_{n}\right|} \geqq n+1, \cdots, \\
& \frac{\left|b_{1}\right|}{2+\left|b_{2}\right|+\left|b_{3}\right|+\cdots+\left|b_{n}\right|} \geqq n+1 \\
& P_{4}^{\prime}(n): b_{i} \text { est du signe de }(-1)^{n+1+i} \text { pour } 1 \leqq i \leqq n
\end{aligned}
$$

Puisque $U$ est un convexe ubiquitaire contenant l'origine, il existe (d'après le Lemme 2.1):

$$
x \in U \text { avec } w+x \in U,
$$

$$
\left.\left.c_{n}^{\prime} \in U \text { avec, pour tout } t \in\right] 0,1\right], 4 e_{n}+t c_{n}^{\prime} \in U \text {. }
$$

Posons $S_{n}=S\left[\left\{e_{1}, e_{2}, \cdots, e_{n}\right\}\right]$.

Puisque $F_{l} \oplus S_{n}$ est de codimension infinie dans $E$ (car $F_{l}$ l'est), il existe un vecteur $g_{l}$, appartenant à la base de départ n'appartenant 
pas à $F_{l} \oplus S_{n}$. Il en résulte qu'il existe:

$$
\lambda \in] 0,1] \text { avec } g_{l^{\prime}}+\lambda c_{n}^{\prime} \notin F_{l} \oplus S_{n} .
$$

Puisque $U$ est un convexe ubiquitaire contenant l'origine, il existe:

(4) $x^{\prime} \in U$ avec, pour tout $\left.\left.\mu \in\right] 0,1\right],-x+\frac{1}{2}\left(g_{l^{\prime}}+\lambda c_{n}^{\prime}\right)+\mu x^{\prime} \in U$.

On a donc, à cause de (1) et (4):

$$
\begin{gathered}
\frac{1}{2}(w+x)+\frac{1}{2}\left(-x+\frac{1}{2}\left(g_{l^{\prime}}+\lambda c_{n}^{\prime}\right)+\mu x^{\prime}\right) \\
=\frac{1}{2} w+\frac{1}{2}\left[\frac{1}{2}\left(g_{l^{\prime}}+\lambda c_{n}^{\prime}\right)+\mu x^{\prime}\right] \in U .
\end{gathered}
$$

Puisque $g_{l^{\prime}}+\lambda c_{n}^{\prime} \notin F_{l} \oplus S_{n}$ (d'après (3)), on peut choisir $\left.\left.\mu \in\right] 0,1\right]$ de façon que

$$
\frac{1}{2}\left(g_{l^{\prime}}+\lambda c_{n}^{\prime}\right)+\mu x^{\prime} \notin F_{l} \oplus S_{n}
$$

On pose alors:

$$
\begin{gathered}
e_{n+1}=-\frac{1}{2}\left[\frac{1}{2}\left(\lambda c_{n}^{\prime}+g_{l^{\prime}}\right)+\mu x^{\prime}\right], \\
f_{n+1}=\frac{1}{2} w-e_{n+1},
\end{gathered}
$$

c'est-à-dire:

$$
a_{n+1, i}=\frac{1}{2} b_{i} \text { pour } 1 \leqq i \leqq n .
$$

$e_{n+1}$ et $f_{n+1}$ satisfont à l'hypothèse de récurrence: En effet $R_{1}(n+1)$ est vérifiée à cause de (1), (4) et (7); $P_{2}(n+1)$ est vérifiée à cause de (6) et (7); $R_{3}(n+1)$ est vérifiée à cause de (5), (7) et (8); $R_{4}(n+1)$ est vérifiée à cause de $P_{3}^{\prime}(n), P_{4}^{\prime}(n)$ et $(9)$.

Montrons que $R_{5}(n+1)$ est vérifiée: Pour tout $\left.\left.t \in\right] 0,1\right]$, on a:

$$
\begin{gathered}
e_{n}-t e_{n+1}=e_{n}+\frac{t}{2}\left[\frac{1}{2}\left(\lambda c_{n}^{\prime}+g_{l^{\prime}}\right)+\mu x^{\prime}\right] \\
=\frac{1}{4}\left(4 e_{n}+t \lambda c_{n}^{\prime}\right)+\frac{1}{4} t g_{l^{\prime}}+\frac{1}{2} t \mu x^{\prime},
\end{gathered}
$$

donc $e_{n}-t e_{n+1} \in U$ à cause de (2) et compte tenu du fait que $\operatorname{tg}_{l^{\prime}} \in U$ et $t \mu x^{\prime} \in U$.

Posons, pour tout $n \in N^{*}, e_{(l, n)}=e_{n}, f_{(l, n)}=f_{n}$ et $c_{(l, n)}=-e_{n+1}$. 
Posons: $E_{l}=S\left[\bigcup_{n \in N^{*}}\left\{e_{(l, n)}\right\}\right]$ et

$$
\left.\left.\Gamma_{2}^{l}=C\left[\bigcup_{n \in N^{*}}\left\{-e_{(l, n)}, f_{(l, n)}, e_{(l, n)}+t c_{(l, n)} \mid t \in\right] 01\right]\right\}\right] .
$$

Puisque, pour tout $n \in N^{*}, R_{4}(n)$ est vraie, il en résulte d'après le 2.1.3 que la famille $\left(f_{(l, n)}\right)_{n \in N^{*}}$ est une base triangulaire de décomposition forte de $E_{l}$ associée à la base $\mathscr{B}_{l}=\left(e_{(l, n)}\right)_{n \in N^{*}}$ de $E_{l}$ telle que, pour tout $n \in \boldsymbol{N}^{*}$, la dernière composante non nulle de $f_{(l, n)}$ dans $\mathscr{B}_{l}$ est $<0$. Puisque, pour tout $n \in N^{*}, c_{(l, n)}=-e_{(l, n+1)}$, il en résulte que $\Gamma_{2}^{l}$ est un convexe ubiquitaire dans $E_{l}$ (c'est même un convexe ubiquitaire-basique de $E_{l}$, d'après le 2.2.2).

Puisque, pour tout $n \in N^{*}, R_{1}(n), R_{3}(n)$ et $R_{5}(n)$ sont vraies, $\Gamma_{2}^{l}$ est contenu dans $U \cap E_{l}$ et ainsi $U \cap E_{l}$ est ubiquitaire.

D'autre part, puisque $e_{(l, 1)}=e_{1}=-g_{l}$ et que, pour tout $n \in N^{*}$, $R_{2}(n)$ est vraie, la somme $\sum_{i \leqq l} E_{i}$ est directe et contient $g_{l}$.

Ainsi $\left(h_{1}\right)$ et $\left(h_{2}\right)$ sont bien vérifiées au rang $l$; notons que pour amorcer la récurrence transfinie, on procède comme précédemment en partant de $F_{i_{0}}=\{0\} \cdot\left(i_{0}\right.$ désigne le plus petit élément de $\left.I\right)$.

La récurrence transfinie est ainsi achevée.

Soit maintenant $\left(E_{j}\right)_{j \in J}$ la sous-famille de la famille $\left(E_{i}\right)_{i \in I}$ composée des $E_{i} \neq\{0\}$. Cette famille vérifie les conclusions du théorème.

\subsection{Théorème de caractérisation.}

ThÉoRÈme 5.2. Soit $E$ un espace vectoriel de dimension infinie.

1. Une condition nécessaire et suffisante pour qu'une partie convexe $U$ de $E$ contenant l'origine soit ubiquitaire est qu'il existe une famille $\left(E_{j}\right)_{j \in J}$ de sous-espaces vectoriels de $E$ telle que l'on ait (i), (ii), (iii) avec:

(i) Pour tout $j \in J, E_{j}$ est de dimension infinie dénombrable.

(ii) La somme des $E_{j}$ est directe et égale à $E$.

(iii) Pour tout $j \in J, U \cap E_{j}$ est sur-ensemble d'un convexe ubiquitaire-basique linéairement borné de $E_{j}$.

2. En conséquence tout convexe ubiquitaire (resp.: convexe ubiquitaire contenant l'origine) $C$ est sur-ensemble d'un convexe ubiquitaire (resp.: convexe ubiquitaire-basique) linéairement borné.

Démonstration. 1. La condition nécessaire résulte du Théorème 5.1, du Théorème 2.4 et du Théorème 3.2.

Montrons que la condition est suffisante.

Désignons, pour tout $j \in J$, par $\Gamma_{2}^{j}$ le convexe ubiquitaire-basique linéairement borné de $E_{j}$ contenu dans $U \cap E_{j}$.

On peut supposer, d'après le Théorème 2.4 , que $\Gamma_{2}^{j}$ s'écrit sous la forme 


$$
\left.\left.\Gamma_{2}^{j}=C\left[\Gamma_{1}^{j} \cup\left(\bigcup_{n \in N^{*}}\left\{e_{(j, n)}+t c_{(j, n)} \mid t \in\right] 0,1\right]\right\}\right)\right],
$$

avec

$$
\Gamma_{1}^{j}=C\left[\bigcup_{n \in N^{*}}\left\{-e_{(j, n)}, f_{(j, n)}\right\}\right],
$$

où $\left(e_{(j, n)}\right)_{n \in N^{*}}$ est une base de $E_{j}$ ( $N^{*}$ étant muni du bon ordre naturel), où $\left(f_{(j, n)}\right)_{n \in N^{*}}$ est une base triangulaire de décomposition forte de $E_{j}$ associée à $\left(e_{(j, n)}\right)_{n \in N^{*}}$ telle que, pour tout $n \in N^{*}$, la dernière composante non nulle de $f_{(j, n)}$ dans la base $\left(e_{(j, n)}\right)_{n \in N^{*}}$ soit $<0$, et où $\left(c_{(j, n)}\right)_{n \in N^{*}}$ est une famille de points de $\Gamma_{1}^{j}$ telle que, pour tout $n \in N^{*}$, la dernière composante non nulle de $c_{(j, n)}$ dans la base $\left(e_{(j, n)}\right)_{n \in N^{*}}$ ait un indice $>n$.

Le Théorème 2.2 montre que $\Gamma_{2}=C\left[\bigcup_{j \in J} \Gamma_{2}^{j}\right]$ est un convexe ubiquitaire-basique de $E$ (qui est linéairement borné d'après le (d) du Théorème 3.1). Puisque $\Gamma_{2} \subset U, U$ est ubiquitaire.

2. Si $C$ contient l'origine, cette deuxième partie est une conséquence de la démonstration qui précède.

Si $C$ ne contient pas l'origine, il suffit d'effectuer sur $C$ une translation qui l'amène à contenir l'origine.

5.5. Remarque. On pourrait montrer la condition suffisante du 1 du Théorème 5.2, sans avoir recours à la notion de convexe ubiquitaire-basique, en utilisant la propriété élémentaire suivante:

Proposition 5.1. Soit, dans un espace vectoriel $E$, une famille $\left(E_{j}\right)_{j \in J}$ de sous-espaces vectoriels telle que $E=\sum_{j \in J} E_{j}$ (la somme n'étant pas nécessairement directe). Si $U$ est une partie convexe de $E$ telle que, pour tout $j \in J, U \cap E_{j}$ est ubiquitaire dans $E_{j}$, alors $U$ est ubiquitaire dans $E$.

Démonstration. Soit $x \in E$; montrons que $x \in \operatorname{lin} U$.

$x$ s'écrit sous la forme $x=\sum_{j \in F} x_{j}$ où $F$ est une partie finie de $J$ et où, pour tout $j \in F, x_{j} \in E_{j}$. Soit $n$ le cardinal de $F$. Puisque, pour tout $j \in J, U \cap E_{j}$ est ubiquitaire dans $E_{j}$, il existe $y_{j} \in U \cap E_{j}$ tel que pour tout $\lambda \in\left[0,1\left[, \lambda n x_{j}+(1-\lambda) y_{j} \in U \cap E_{j}\right.\right.$.

Il en résulte que, pour tout $\lambda \in[0,1[$,

$$
\lambda x+(1-\lambda) \sum_{j \in F} \frac{1}{n} y_{j}=\frac{1}{n} \sum_{j \in F}\left(\lambda n x_{j}+(1-\lambda) y_{j}\right) \in U,
$$

donc que $x \in \operatorname{lin} U$.

Nous remercions le Referee pour l'étude détaillée de notre article et pour ses aimables suggestions. 


\section{REFERENCES}

1. G. Coquet, Sur les familles de décomposition et leurs applications à la théorie des ensembles convexes, Thèse de Doctorat d'Etat, $n^{\circ}$ d'ordre 287, Université des Sciences et Techniques de Lille, 1973.

2. G. Coquet et J. C. Dupin, Décomposition d'un espace vectoriel de dimension infinie en une somme de deux convexes, C. R. Acad. Sc. Paris, série A, 274 (1972), 899-902.

3. - Une caractérisation des convexes ubiquitaires, C. R. Acad. Sc. Paris, série A, 274 (1972), 1167-1169.

4. J. C. Dupin et G. Coquet, Convexes ubiquitaires et convexes ubiquitaires linéairement bornés, C. R. Acad. Sc. Paris, série A, 278 (1974), 613-615.

5. V. Klee, Convex sets in linear spaces, Duke Math. J., 18 (1951), 443-466.

6. - Convex sets in linear space III, Duke Math, J., 20 (1953), 105-112.

Received June 12, 1974.

Centre Universitaire de Valenciennes, France 


\title{
PACIFIC JOURNAL OF MATHEMATICS
}

\author{
EDITORS
}

RICHARD ARENS (Managing Editor)

University of California

Los Angeles, California 90024

R. A. Beaumont

University of Washington

Seattle, Washington 98105
J. DugundJI

Department of Mathematics University of Southern California Los Angeles, California 90007

D. Gilbarg and J. Milgram

Stanford University

Stanford, California 94305

\section{ASSOCIATE EDITORS}

E. F. BECKENBACH

B. H. NeUManN

F. WOLF

K. YosHIDA

\section{SUPPORTING INSTITUTIONS}

UNIVERSITY OF BRITISH COLUMBIA

GALIFORNIA INSTITUTE OF TECHNOLOGY

UNIVERSITY OF CALIFORNIA

MONTANA STATE UNIVERSITY

UNIVERSITY OF NEVADA

NEW MEXICO STATE UNIVERSITY

OREGON STATE UNIVERSITY

UNIVERSITY OF OREGON

OSAKA UNIVERSITY
UNIVERSITY OF SOUTHERN CALIFORNIA

STANFORD UNIVERSITY

UNIVERSITY OF TOKYO

UNIVERSITY OF UTAH

WASHINGTON STATE UNIVERSITY

UNIVERSITY OF WASHINGTON

AMERICAN MATHEMATICAL SOCIETY NAVAL WEAPONS CENTER 


\section{Pacific Journal of Mathematics}

\section{Vol. 57, No. $1 \quad$ January, 1975}

Keith Roy Allen, Dendritic compactification ......................... 1

Daniel D. Anderson, The Krull intersection theorem ..................... 11

George Phillip Barker and David Hilding Carlson, Cones of diagonally dominant

matrices .............................................

David Wilmot Barnette, Generalized combinatorial cells and facet splitting ........

Stefan Bergman, Bounds for distortion in pseudoconformal mappings ............

Nguyên Phuong Các, On bounded solutions of a strongly nonlinear elliptic

equation ...............................................

Philip Throop Church and James Timourian, Maps with 0-dimensional critical

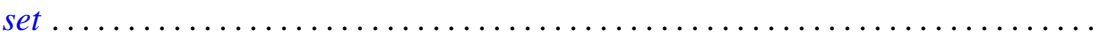

G. Coquet and J. C. Dupin, Sur les convexes ubiquitaires ................. 67

Kandiah Dayanithy, On perturbation of differential operators............... 85

Thomas P. Dence, A Lebesgue decomposition for vector valued additive set

functions .............................................. 91

John Riley Durbin, On locally compact wreath products.................. 99

Allan L. Edelson, The converse to a theorem of Conner and Floyd ............. 109

William Alan Feldman and James Franklin Porter, Compact convergence and the

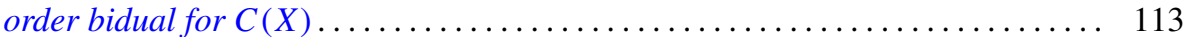

Ralph S. Freese, Ideal lattices of lattices......................... 125

R. Gow, Groups whose irreducible character degrees are ordered by divisibility ... 135

David G. Green, The lattice of congruences on an inverse semigroup ............ 141

John William Green, Completion and semicompletion of Moore spaces .......... 153

David James Hallenbeck, Convex hulls and extreme points of families of starlike and close-to-convex mappings .................................. 167

Israel (Yitzchak) Nathan Herstein, On a theorem of Brauer-Cartan-Hua type ...... 177

Virgil Dwight House, Jr., Countable products of generalized countably compact spaces ............................................ 183

John Sollion Hsia, Spinor norms of local integral rotations. I . . . . . . ......... 199

Hugo Junghenn, Almost periodic compactifications of transformation

semigroups....................................

Shin'ichi Kinoshita, On elementary ideals of projective planes in the 4-sphere and oriented $\Theta$-curves in the 3 -sphere ............................ 217

Ronald Fred Levy, Showering spaces............................ 223

Geoffrey Mason, Two theorems on groups of characteristic 2-type............. 233

Cyril Nasim, An inversion formula for Hankel transform ................... 255

W. P. Novinger, Real parts of uniform algebras on the circle ................ 259

T. Parthasarathy and T. E. S. Raghavan, Equilibria of continuous two-person

games............................................... 265

John Pfaltzgraff and Ted Joe Suffridge, Close-to-starlike holomorphic functions of several variables ....................................... 271

Esther Portnoy, Developable surfaces in hyperbolic space .................. 281

Maxwell Alexander Rosenlicht, Differential extension fields of exponential type . . . 289

Keith William Schrader and James Lewis Thornburg, Sufficient conditions for the

existence of convergent subsequences ........................... 301

Joseph M. Weinstein, Reconstructing colored graphs .................. 307 